\title{
Estudios en las Apocynaceae Neotropicales: nuevas especies de Lacmellea (Rauvolfioideae, Willughbeeae) para Sur América
}

\author{
JUAN FRANCISCO MORALES ${ }^{1}$
}

(recebido: 15 de setembro de 2005; aceito: 15 de março de 2007)

\begin{abstract}
Studies in the Neotropical Apocynaceae: new species of Lacmellea (Rauvolfioideae, Willughbeeae) from South America). Lacmellea bahiensis J.F. Morales and L. macrantha J.F. Morales, two new species from South America are described. Lacmellea bahiensis is endemic to the state of Bahia, Brazil and has been confused with L. aculeata (Ducke) Monach., but differs by its smaller corollas and sepals. Lacmellea bahiensis is also similar to L. pauciflora (Kuhlm.) Markgr., but differs by its longer corolla tube (21-23 mm vs. 15-17 mm) and wider leaves $((2,5-) 3,5-5,5 \mathrm{~cm} v s .1,4-2,2(-3,1) \mathrm{cm})$. Lacmellea macrantha, known from Ecuador and Peru, has been confused with L. floribunda (Poepp.) Benth. and L. speciosa Woodson, but $L$. macrantha differs from the first taxon by its larger floral bracts, wider sepals, and longer corolla lobes and from the second one, by its larger corollas, longer corolla lobes and bigger fruits. Illustrations, discussion of their taxonomic affinities and specimens examined are included.
\end{abstract}

Key words - Apocynaceae, Lacmellea bahiensis, Lacmellea macrantha, Neotropics, new species, Rauvolfioideae

RESUMEN - (Estudios en las Apocynaceae Neotropicales: nuevas especies de Lacmellea (Rauvolfioideae, Willughbeeae) para Sur América). Se describen Lacmellea bahiensis J. F. Morales y L. macrantha J. F. Morales, dos nuevos taxones para Sur América. Lacmellea bahiensis es endémica a Bahia, Brasil y ha sido confundida con L. aculeata (Ducke) Monach., de la que difiere por sus corolas y sépalos más pequeños. De L. pauciflora (Kuhlm.) Markgr., L. bahiensis difiere por sus flores con el tubo de la corola más largo (21-23 mm vs. 15-17 mm) y hojas usualmente más anchas $((2,5-) 3,5-5,5 \mathrm{~cm} v s .1,4-2,2(-3,1) \mathrm{cm})$. Lacmellea macrantha, conocida de Ecuador y Perú, se ha confundido con L. floribunda (Poepp.) Benth. y L. speciosa Woodson, pero difiere de la primera por sus brácteas florales más grandes, sépalos más anchos y lóbulos de la corola más largos, y de la segunda por corolas más largas, con los lóbulos más largos y sépalos más anchos y frutos más grandes. Ilustraciones, discusión de sus afinidades taxonómicas y listado de especímenes examinados son incluidos para cada una de ellas.

Palabras clave - Apocynaceae, Lacmellea bahiensis, Lacmellea macrantha, Neotrópicos, nuevas especies, Rauvolfioideae

\section{Introducción}

Lacmellea (Apocynaceae, Rauvolfioideae) es el género más grande de la tribu Willughbeeae (sensu Endress \& Bruyns 2000) en el neotrópico, con cerca de 22 taxones conocidos. La taxonomía del género no es sencilla, debido principalmente a que la mayoría de las especies son idénticas vegetativamente y ante la ausencia de flores y la presencia de frutos inmaduros, algunos taxones pueden ser difíciles de separar. El género necesita urgentemente una revisión, debido a que en las monografías de Markgraf(1941) y Monachino (1944), varias especies eran conocidas por muy pocas colecciones o en su defecto, no se conocían buenas colecciones con flores (un carácter con relativa importancia dentro del género), por lo que en algunos casos se manejaron conceptos de taxones muy amplios e incluyentes o bastante

1. Instituto Nacional de Biodiversidad (INBio), Apartado Postal 22-3100, Santo Domingo, Heredia, Costa Rica, fmorales@inbio.ac.cr reducidos. Así mismo, el número de especies aceptadas en ambos trabajos es algo divergente, pues mientras Markgraf solo aceptó 14 especies, Monachino incluyó un total de 18 taxones. Adicionalmente, en conjunción con la descripción de L. costanensis, Steyermark (1964) cuestionó la utilidad de algunos de los caracteres (e.g., ápice de las anteras, pubescencia de la cabeza estigmática) utilizados por Monachino (1944) en su clave, ya que un análisis preliminar de los mismos en algunos taxones, demostró que no son consistentes. En los últimos 60 años, con la excepción de una breve sinopsis para las especies del género en Mesoamérica, que incluyó la descripción de una nueva especie (Morales 1998), no se ha realizado ningún trabajo monográfico (excluyendo tratamientos locales de floras) y pocas especies adicionales han sido descritas (i.e., Monachino 1958, 1961, Steyermark 1964, Woodson 1949).

Como resultado del estudio de colecciones de Lacmellea para una nueva sinopsis del género y para diversos proyectos florísticos en Sur América, se encontraron dos nuevas especies. La primera de ellas está restringida al E de Brasil, en el Estado de Bahia, 
un área geográfica donde solamente se conoce otra especie del género (i.e., Lacmellea pauciflora (Kuhlm.) Markgr.). La segunda nueva especie, ha sido comúnmente confundida con Lacmellea floribunda (Poepp.) Benth. y L. speciosa Woodson de las que difiere principalmente por el tamaño de las flores y frutos. Ambas especies se describen a continuación y se discuten sus relaciones con las especies afines. Las medidas de partes vegetativas o florales de otros taxones citados en el texto han sido tomadas del borrador de una nueva sinopsis del género (J. F. Morales, datos sin publicar), por lo que pueden diferir de las citadas por Markgraf (1941) o Monachino (1944).

\section{Resultados y Discusión}

Lacmellea bahiensis J. F. Morales, sp. nov.

Tipo: BRASIL. BAHIA: Una, Reserva Biológica do Mico-leão (IBAMA), 12-XII-1995 (fl), A. Carvalho et al. 6180 (Holotipo CEPEC; Isotipos MO, NY).

Figura 1

A Lacmellea aculeata (Ducke) Monach., cui similis, corollae tubis 21-23 mm longis (vs. 27-34 mm), et calycis lobis 1,4-2,0 mm longis (vs. 2,2-3,5 mm) differt; a L. pauciflora (Kuhlm.) Markgr. affinis, corollae tubis 21-23 mm longis (vs. 15-17 mm) et foliis (2,5-)3,5-5,5 cm latis (vs. 1,4-2,2(-3,1)) differt.

Árboles o lianas (?) de 4 a $30 \mathrm{~m}$ alt., el tronco sin aguijones, ramitas aplanadas cuando jóvenes, subteretes con la edad, glabras. Hojas con el pecíolo 6-10 mm long., glabro; láminas $(6,5) 7,8-15,5(-17,2)$ x (2,5-)3,5-5,5 cm, elípticas a ovado-elípticas, el ápice agudo o cortamente apiculado, la base obtusa a redondeada, raramente levemente subcordada, glabras, sin puntuaciones negras abaxialmente, los márgenes a veces levemente revolutos, venas secundarias separadas por $4-10 \mathrm{~mm}$ entre sí. Inflorescencia axilar, raramente terminal, usualmente con (1-)3-4 flores, glabra, pedúnculo 2-12 mm long., pedicelos 5-11 mm long., brácteas 1,0-1,5 x 0,5-1,0 mm, bractéolas $0,8-1,5 \times 0,8-1,2 \mathrm{~mm}$; sépalos 1,4-2,0 × 2,4-4,0 $\mathrm{mm}$, anchamente ovados, redondeados apicalmente, glabrescentes, pero usualmente diminutamente ciliolados en el margen; corola blanca, glabra exteriormente, pero el tubo internamente pubescente, tubo 21-23 x 2,5-5,0 $\mathrm{mm}$, abultado en la base y en la posición de los estambres; lóbulos 5,0-5,5 x 3,0-4,0 mm, ovados; anteras 5,2-6,0 mm long., dorsalmente glabras, cabeza estigmática $2,5-3,0 \mathrm{~mm}$ long., ovario 3,5-5,5 mm long. Frutos globosos a subglobosos, 1,7-2,3 cm diám., amarillos al madurar.

Paratipos: BRASIL. BAHIA: Cairo, camino a Povoados de Torrinha y Tapuia, 25-X-1984, L. Silva \&
T. Dos Santos 1773 (CEPEC, WAG); Ilhéus, Río Fundão, 12-I-1995, A. Carvalho et al. 5425 (CEPEC, MO), 13-I-199, A. Carvalho et al. 5561 (CEPEC, MO, NY); Ilhéus, al SO de Olivença, camino a Vila Brasil, 21-V-1985, W. Thomas et al. 10941 (WAG); Itacare, Marahu, Camamu and Canavieiras, 1946, G. Bondar 10 (US); Una, Reserva Ecológica do Mico-leão, IV1993, A. Amorin et al. 1229 (CEPEC, NY); Una, Maruim, límites de Fazendas Maruim y Dois de Julho, SO de Olivença, 30-IV-1981, S. Mori et al. 13849 (CEPEC, MO, NY, WAG); entre Una y Olivença, 17VI-1971, R. Pinheiro 1397 (CEPEC, WAG); Una, estación experimental Lemos Maia, 17-VI-1980, $A$. Rylands s.n. (CEPEC, WAG); Uruçuca, 26-VI-1979, S. Mori 12049 (CEPEC, MO, NY, WAG).

Distribución, hábitat y fenología: Endémica al Estado de Bahia, en Brasil, donde crece en bosques muy húmedos, en elevaciones de 0-200 m. Especímenes con flores han sido recolectados entre diciembre y febrero. Material con frutos se conoce de enero, febrero y de abril a junio.

Nombres comunes: Chananão (Brasil, Bahia, Pontal de Itacaré, Marahu); chananã (Brasil, Bahia, Torrinha y Tapuia); Chinené (Brasil, Bahia, Una).

Lacmellea bahiensis es una especie muy notable, que se puede reconocer con facilidad por la siguiente combinación de caracteres: láminas foliares usualmente con el margen algo involuto, inflorescencias reducidas a 3 ó 4 flores, flores con los sépalos 2,4-4,0 mm de ancho y corola con el tubo de 21-23 mm longitud. Al igual que la mayoría de especies del género, L. bahiensis carece de puntuaciones negras en la superficie abaxial de las hojas. Lacmellea bahiensis también ha sido confundida con $L$. aculeata (Ducke) Monach., de la que difiere el tubo de la corola más pequeño (21-23 mm vs. 27-34 mm), sépalos más cortos $(1,4-2,0 \mathrm{~mm}$ vs. 2,2-3,5 mm) y distribución geográfica disyunta, ya que $L$. bahiensis es endémica al E del Estado de Bahia, mientras que L. aculeata, es un taxón restringido a la cuenca baja amazónica. Lacmellea bahiensis representa el segundo taxón conocido del Estado de Bahia en Brasil; la otra especie conocida de la misma región (L. pauciflora (Kuhlm.) Markgr.), tiene flores con el tubo de la corola inferior a $17 \mathrm{~mm}$ long. (vs. 21-23 mm), así como láminas foliares más angostas (1,4$2,2(-3,1) \mathrm{cm} v s .(2,5-) 3,5-5,5 \mathrm{~cm})$.

Hasta el momento se han registrado dos tipos de hábito diferentes para esta especie: algunas colecciones indican que es una liana o arbusto escandente, (e.g., Carvalho et al. 6180; Mori et al. 13849), mientras que la gran mayoría indican que es un árbol (e.g., Amorin et al. 1229). Son necesarios más estudios de 


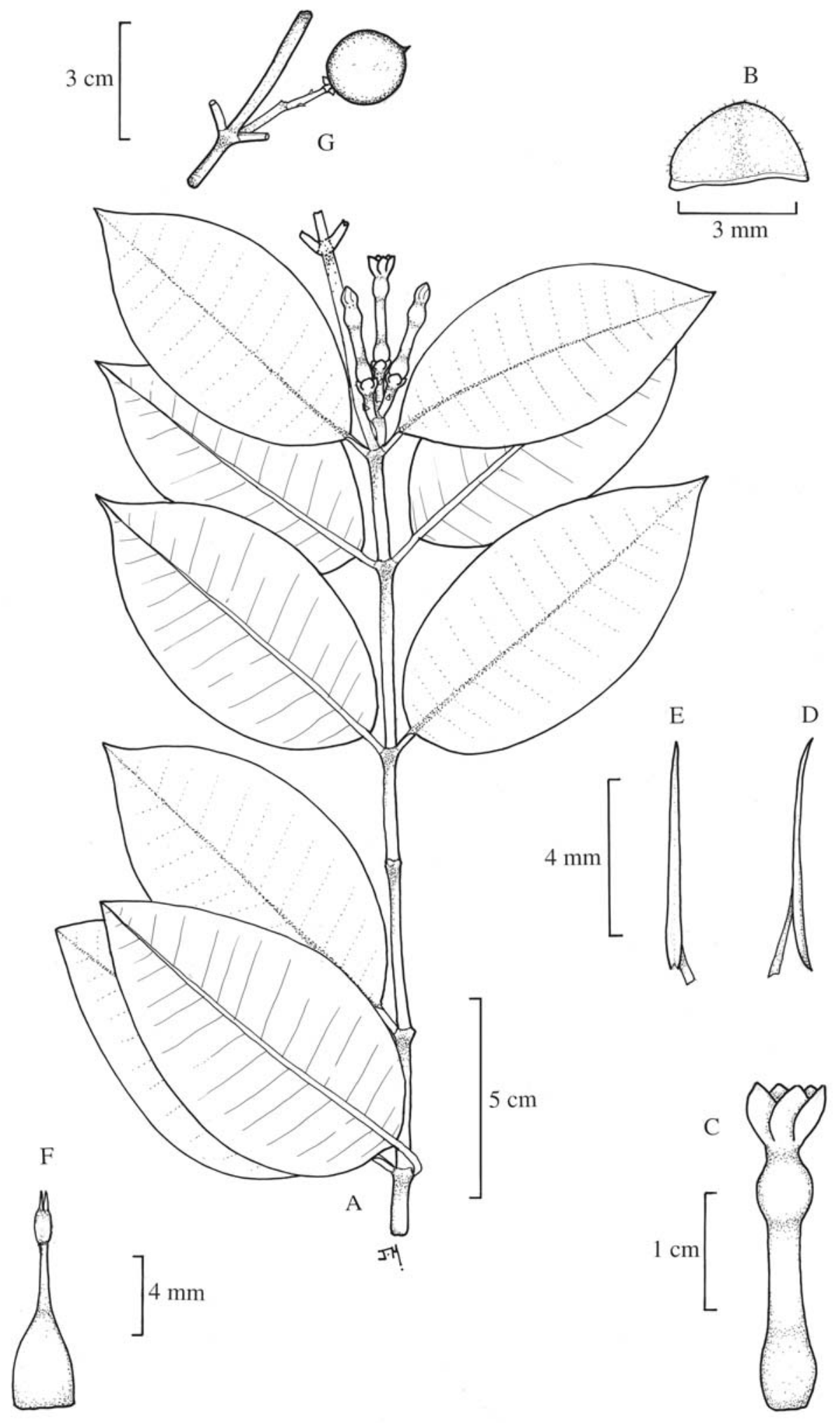

Figura 1. Lacmellea bahiensis J. F. Morales A. Ramita con inflorescencias. B. Vista de la cara abaxial de un sépalo. C. Detalle del tubo de la corola. D. Antera, vista lateral. E. Antera, vista dorsal. F. Gineceo. G. Fruto. (A-F Carvalho et al. 6180, MO; G Mori et al. 13849, WAG).

Figure 1. Lacmellea bahiensis J. F. Morales A. Flowering branch. B. Adaxial surface of one sepal. C. Corolla tube. D. Anther, lateral view. E. Anther, dorsal view. F. Gynoecium. G. Fruit. (A-F Carvalho et al. 6180, MO; G Mori et al. 13849, WAG). 
campo para determinar con certeza el hábito real de Lacmellea bahiensis. El epíteto hace referencia al estado de Bahia en Brasil, único sitio de donde es conocido este taxón.

Lacmellea macrantha J. F. Morales, sp. nov. Tipo: ECUADOR. NAPO: Río Napo, Reserva Biológica Jatun Sacha, 25-I-1986 (fl, fr), W. Palacios \& D. Neill 973 (Holotipo QCNE; Isotipos MO, NY, QCA, WAG). Figura 2.

$A$ Lacmellea floribunda (Poepp.) Benth., cui similis, bracteis (2,4-)3-4,5 mm longis (vs. 0,5-1,5 mm), calycis lobis 3,0-3,6 mm latis (vs. 1,5-2,0 mm), corollae lobis 6,8-8,0 mm longis (vs. 3,0-4,5 mm) et fructus $4-5 \mathrm{~cm}$ longis (vs. 1,6-1,8 cm) differt; a L. speciosa Woodson cui affinis, corollae tubis 37-41 mm longis (vs. 23-30 $\mathrm{mm}$ ), lobis 6,8-8,0 mm longis (vs. 11-14 mm), et fructus 4,0-5,0 cm longis (vs. 2,5-3,5 cm) differt.

Árboles 6-15(-25) m alt., el tronco aparentemente sin aguijones, ramitas conspicuamente aplanadas cuando jóvenes, subteretes con la edad, glabras o glabrescentes. Hojas: pecíolo 10-14 mm long., glabro o glabrescente; láminas (10,5-)11,5-23,4 x 4,2-11,1 cm, anchamente elípticas, el ápice agudo o cortamente acuminado, la base obtusa o anchamente cuneada, glabras o glabrescentes, con puntuaciones negras muy esparcidas abaxialmente, sobretodo a lo largo del nervio central y en el tercio basal, usualmente solo visibles con aumento, no revolutas marginalmente, venas secundarias separadas por 6-15 mm entres sí. Inflorescencia axilar, con 4 a 9 flores, inconspicuamente puberulenta a glabrescente, pedúnculo 7-18 $\mathrm{mm}$ long., pedicelos 2-4 $\mathrm{mm}$ long. brácteas (2,4-)3,0-4,5 mm long., bractéolas 1,2-2,2 mm long.; sépalos 2,1-3,3 x 3,0-3,6 mm, anchamente ovados, redondeados apicalmente, esparcida y diminutamente puberulentos a glabrescentes, diminutamente ciliolados en el margen, aunque a veces los cilios muy esparcidos; corola con el tubo verde y los lóbulos blancos, la superficie abaxial de los lóbulos inconspicuamente papilado-puberulenta, tubo $37-41 \times 2,2-5,0 \mathrm{~mm}$, abultado en la base y en la posición de las anteras; lóbulos 6,8-8,0 x 3,0-3,4 mm, angostamente ovado-elípticos; anteras 8,5-9,0 mm long., glabras dorsalmente, cabeza estigmática 2,4-2,8 mm long., ovario 2-3 mm long. Frutos suborbiculares a anchamente elípticos, 4-5 cm long., amarillos o anaranjados al madurar.

Paratipos: ECUADOR. Esmeraldas: Quininde, montañas Mache, Reserva Ecológica Mache-Chindul, 30-III-1996, J. Clark et al. 2350 (MO, NY, QCNE, US, WAG); Quininde, Estación Biológica Bilsa, montañas Maché, O de Santa Isabel, 15-VII-1996, J. Clark et al. 2883 (INB, MO, QCNE, US, WAG). NAPO: debajo de Misahualli, Río Napo, 13-X-1985, D. Neill \& Priest 6920 (MO, NY, QCA, QCNE, WAG); Reserva Biológica Jatún Sacha, Río Napo, 1-15-IX-1987, W. Palacios 1913 (QCNE); camino de los Aucas, Ahuano, 9-VI-1991, M. Ríos \& Vivanco 393 (MO, NY, QCA). Morona-Santiago: Puerto Morena, NE de Río Morona, 30-IX / 2-X-1975, E. Little 575 (Q, QCNE); Taisha, 15-X-1975, E. Little et al. 715 (COL, Q, US); N de Taisha, 20-23-VIII-1976, A. Ortega 83 (Q). Pastaza: Pastaza, pozo petrolero Masaramu, V1990, E. Gudiño 408 (MO, QCNE). PERÚ. LORETO: Maynas, Sucusari, 7-X-1986, R. Vásquez \& J. Jaramillo 8218 (MO, WAG).

Distribución, hábitat y fenología: Conocida principalmente en el SE de Ecuador y en Perú, donde crece en bosques muy húmedos, en elevaciones de 300$500 \mathrm{~m}$. Especímenes con flores y frutos han sido recolectados entre septiembre y octubre.

Lacmellea macrantha ha sido confundida con $L$. floribunda (Poepp.) Benth. y L. speciosa Woodson, grupo de taxones que se caracterizan por sus hojas con láminas foliares relativamente grandes, así como corolas con el tubo de más de $23 \mathrm{~mm}$ de longitud. Lacmellea macrantha difiere de L. speciosa por la mayor longitud del tubo de la corola $(37-41 \mathrm{~mm} v s$. 23-30 mm), lóbulos más pequeños (6,8-8,0 mm vs. 11-14 $\mathrm{mm})$, así como sépalos mucho más grandes (2,1-3,3 x $3,0-3,6 \mathrm{~mm} v \mathrm{~s} .1-1,5 \times 1,5-2 \mathrm{~mm}$ ) y frutos más grandes (4-5 cm vs. 2,5-3,5 cm). Lacmellea floribunda se puede diferenciar de L. macrantha por sus láminas foliares sin puntacuaciones negras abaxialmente (vs. puntuaciones negras esparcidas presentes, sobretodo a lo largo del nervio central y en el tercio basal), brácteas florales más pequeñas $(0,5-1,5 \mathrm{~mm} v s .(2,4-) 3,0-4,5$ $\mathrm{mm})$, sépalos más angostos $(1,5-2,0 \mathrm{~mm}$ vs. 3,0-3,6 $\mathrm{mm})$, lóbulos de la corola más pequeños $(3,0-4,5 \mathrm{~mm}$ vs. $6,8-8,0 \mathrm{~mm})$ y frutos mucho más pequeños $(1,6-1,8$ $\mathrm{cm} v s .4-5 \mathrm{~cm}$ ). El epíteto se refiere al tamaño de las flores, las más grandes conocidas en el género hasta ahora.

Agradecimientos - Se agradece a los siguientes herbarios por permitir el uso y acceso de sus colecciones: CEPEC, COL, MO, MOL, NY, Q, QCA, QCNE, U, US, USM, WAG. También quiero agradecer a las siguientes personas por facilitar el acceso a diferentes herbarios o brindar facilidades logísticas para la visita de los mismos: Julio Betancur (COL), Asunción Cano(USM), José Luis Fernández-Alonso (COL), Paul e Hiltje Maas (U), Rocío Rojas (HOXA) y Homero Vargas (QCNE). 


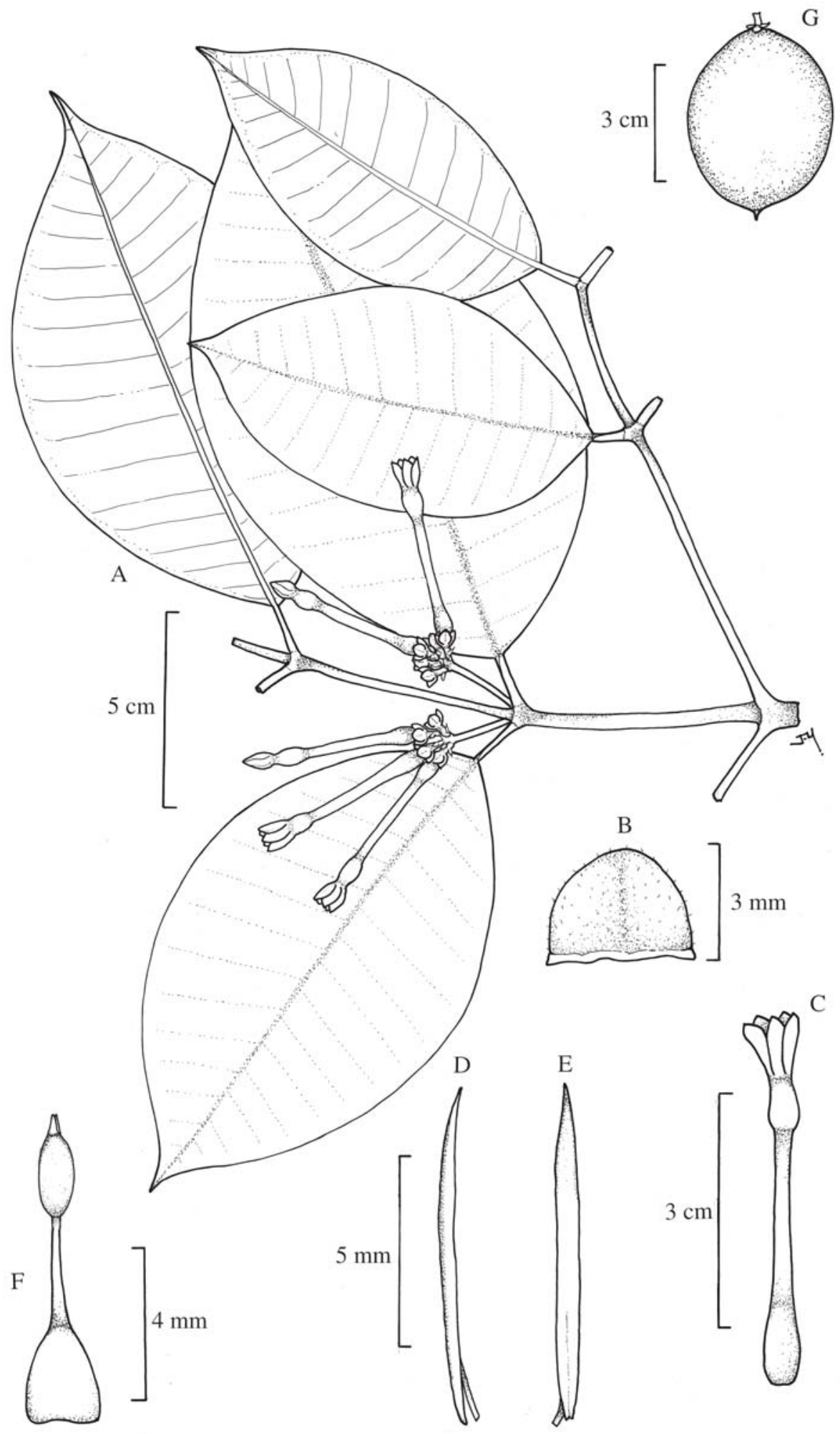

Figura 2. Lacmellea macrantha J. F. Morales A. Ramita con inflorescencias. B. Vista de la cara abaxial de un sépalo. C. Detalle del tubo de la corola. D. Antera, vista lateral. E. Antera, vista dorsal. F. Ovario y porción basal del estilo. G. Fruto. (A-F Palacios \& Neill 973, MO; G Neill \& Priest 6920, MO).

Figure 2. Lacmellea macrantha J. F. Morales A. Flowering branch. B. Sepal, adaxial surface. C. Corolla tube. D. Anther, lateral view. E. Anther, dorsal view. F. Gynoecium. G. Fruit. (A-F Palacios \& Neill 973, MO; G Neill \& Priest 6920, MO). 


\section{Referencias bibliográficas}

ENDRESS, M. E. \& BRUYNS, P. 2000. A revised classification of the Apocynaceae s.l. The Botanical Review 66:1-56.

MARKGRAF, F. 1941. Die gattung Lacmellea H. Karsten. Notizblatt des Botanischen Gartens und Museums zu Berlin-Dahlem 15:615-629.

MONACHINO, J. 1944. A Revision of Lacmellea and the transfer of Zschokkea (Apocynaceae). Lloydia 7:275-302.

MONACHINO, J. 1958. Apocynaceae. In The Botany of the Guyana Highland III (B. Maguire \& J. Wurdack, eds.). Memoirs of the New York Botanical Garden 10:117-138.
MONACHINO, J. 1961. Apocynaceae. In The Botany of the Guyana Highland IV (B. Maguire \& J. Wurdack, eds.). Memoirs of the New York Botanical Garden 10:58-65.

MORALES, J.F. 1998. Sinopsis del género Lacmellea (Apocynaceae) en Mesoamérica, con una nueva especie de Costa Rica. Novon 8:259-262.

STEYERMARK, J. 1964. New species of cow tree (Lacmellea) from Distrito Federal, Venezuela. Boletín de la Sociedad Venezolana de Ciencias Naturales 25:240-244.

WOODSON, R. 1949. New Apocynaceae of South America. Annals of the Missouri Botanical Garden 36:543-546. 